\title{
Triple-negative and HER2-overexpressing breast cancer cell sialylation impacts tumor microenvironment T-lymphocyte subset recruitment: a possible mechanism of tumor escape
}

This article was published in the following Dove Press journal: Cancer Management and Research

\author{
Christian Garbar ${ }^{1,2}$ \\ Corinne Mascaux ${ }^{1,2}$ \\ Yacine Merrouche $e^{1,2}$ \\ Armand Bensussan ${ }^{3}$ \\ 'Biopathology Department, Institut \\ Jean Godinot - Unicancer, Reims, \\ France; ${ }^{2}$ DERM-I-C EA7319, \\ Université de Reims Champagne - \\ Ardenne, Reims, France; ' 3 INSERM \\ U976; Université Paris Diderot, \\ Sorbonne Paris Cité, Laboratory \\ of Immunology, Dermatology \& \\ Oncology, Paris, France
}

\begin{abstract}
Introduction: Breast cancers develop different patterns of sialylation to modulate their tumor-infiltrating lymphocyte (TIL) environment. We studied the relationship between $\alpha-2,6$ sialyltransferases and the TIL in different breast cancer molecular subgroups.

Materials and methods: Immunohistochemical preparations were made from 39 luminal (LUM), 13 human epidermal growth factor receptor 2-overexpressing (HER2) and 47 triplenegative (TN) breast carcinomas. Targeted proteins included ST6Gal-I, ST6Gal-II, ST6GalNac-I, CD8, CD4 and granzyme-B in both cytotoxic T lymphocytes and NK lymphocytes (CTL/NK). Results: CTL/NK populations were significantly more frequent in TN than LUM $(P<0.001)$. TN showed a lower level of ST6Gal-I expression than LUM or HER2 (both $P>0.001$ ). ST6GalNac-I expression was lower in LUM than in TN or HER2 $(P=0.002$ and $P=0.02$, respectively). In HER2, a significant association was found between a low level of ST6Gal-I expression and a high TIL level. In TN, a significant association was observed between a high level of ST6Gal-II expression and a high TIL level.

Conclusion: An increase in infiltrating lymphocytes could be influenced by low expression of ST6Gal-I in HER2 and by high expression of ST6Gal-II in TN breast cancers. Thus, targeting these sialylation pathways could modulate the levels of TIL.
\end{abstract}

Keywords: breast, carcinoma, sialyltransferase, triple-negative, HER2

\section{Introduction}

To date, the molecular classification of breast cancers (BCs) proposed by Perou et al has been categorized according to the targeted therapy against these tumors. These authors have described three main molecular subtypes: luminal (LUM) BCs that express hormonal estrogen and progesterone receptors $(\mathrm{ER}+$ and $\mathrm{PR}+$ ) but no human epidermal growth factor receptor 2 (HER2-), overexpressed HER2 BC (HER2; phenotype: ER \pm PR \pm HER2+) and triple-negative (TN; phenotype: ER-/PR-/HER2-). ${ }^{1}$

It is now well established that tumor-infiltrating lymphocytes (TILs) play an important role in chemotherapy and that their stimulation or inhibition can influence the tumor therapeutic response and patient prognosis., ${ }^{2,3}$ However, cell interactions between cancer cells and the TIL are complex, and our present knowledge represents only the tip of the iceberg. One of these intercellular communication mechanisms
Correspondence: Christian Garbar; Armand Bensussan

Biopathology Department, Institut Jean Godinot - Unicancer, I rue du Général Koenig CS800I4, 51726 Reims, France. Tel: +33326504267

Email: christian.garbar@reims.unicancer. fr; corinne.garbar-mascaux@reims. unicancer.fr 
likely consists of changes to the cytoplasmic membrane, particularly in glycoproteins. In cancer cells, the most important glycoprotein alteration is sialylation. ${ }^{4}$

Sialylation consists of adding sialic acids (Sia) on an $N$ - or $O$-linked glycoprotein. Sialylation is regulated by a balance of two enzyme families: the sialyltransferases, which add Sia, and the sialidases (or the neuraminidases, Neu), which remove Sia. Sialyltransferases are a family of $\sim 20$ different enzymes linking Sia to a galactose (Gal) in an $\alpha-2,3$ position (ST3Gal)or an $\alpha-2,6$ position (ST6Gal) or to an $N$-acetylgalactosamine (ST6GalNac). The third sialyltransferase family (ST8) promotes $\alpha-2,8$ linkage. Four sialidases remove Sia residues: Neu1, localized in lysosomes and the cell surface; Neu2 or Neu4, located in the cytosol and Neu3, located in the cell membrane. ${ }^{5}$

Alpha-2,6 sialylation is the most commonly observed sialylation mechanism in tumor cells and is associated with an upregulation of both ST6Gal-I and ST6GalNac-I sialyltransferases. ${ }^{6}$ Two of the most well-characterized substrates of these sialyltransferases are MUC1 and EGFR. Moreover, Wreschner et al demonstrated an association between MUC1 and EGFR. ${ }^{7}$ Interestingly, Lillehoj et al suggested that both glycoproteins could be associated with $\mathrm{Neu}$, one of the desialylation enzymes. ${ }^{8}$ These authors suggested that Neu1 could regulate EGFR and MUC1 signaling. ${ }^{7,8}$ We recently demonstrated that Neul and MUC1 have reduced expression in TN compared to LUM tumors, and that EGFR is more expressed in TN than LUM tumors, suggesting that the EGFR-MUC1Neu1 molecular pathway is complex. ${ }^{9}$ Intriguingly, in breast cancer, EGFR has been observed to be highly sialylated, and these changes have been shown to influence its metabolic activities and induce chemoresistance. ${ }^{10}$

Here, we investigated whether TILs and malignant cell sialylation were associated, and we discussed the possibility that this plays a role in the development of resistance to chemotherapy or targeted therapy.

To understand the $\alpha-2,6$-sialylation pathway and its relationship with TIL, we determined the expression of three $\alpha$-2,6-sialylidases (ST6Gal-I, ST6Gal-II and ST6GalNac-I), as well as their relationship with different TIL subsets, including CD4, CD8 and CTL/NK cells (granzyme B-positive cells; cytotoxic T/natural killer lymphocytes, respectively), in different molecular breast cancer groups.

\section{Materials and methods Patient population}

Archival paraffin-embedded surgical material and clinical data from 99 women presenting with breast cancer were available for this study. All cases were classified according to immunohistochemical classification by means of a preliminary immunohistochemical study and subsequent confirmation by tissue microarray (TMA). Our data set included 39 luminal carcinomas (LUM, age $=59.3 \pm 12.9$ years), 13 HER2 over-expressing carcinomas (HER2, age $=58.1 \pm 13.1$ years $)$ and $47 \mathrm{TN}$ carcinomas (age $=61.7 \pm$ 14.5 years, $P=$ ns). No neo-adjuvant chemotherapy was performed.

This study was performed with the approval of the local ethics committee (Centre de Resources Biologiques de l'Intitut Jean Godinot) and conformed to the Declaration of Helsinki. Written informed consent was provided by all participants and all patients were informed and agreed to contribute to this study.

\section{Histological procedures and Tissue Micro Array (TMA) construction}

All surgical specimens were initially fixed in $4 \%$ buffered formaldehyde solution for $8-48 \mathrm{~h}$, then embedded in paraffin and cut into 4- $\mu$ m-thick sections. From these archival formaldehyde/paraffin blocks, we built a TMA to receive a paraffin block to perform all immunohistochemical studies. We used an automated TMA device, Minicore2 (Excilone, Elancourt, France) associated with a needle core $0.6 \mathrm{~mm}$ in diameter. We chose 3 distant core needle samples of each donor tumor paraffin block.

\section{Immunohistochemical methods}

Immunohistological staining was performed with a Dako Autostainer Link 48® immunostaining system (Dako Denmark A/S, Glostrup, Denmark). After dewaxing, antigenic retrieval was performed using citrate-buffered $(\mathrm{pH} 6)$ or EDTA-buffered ( $\mathrm{pH} 9$ ) antigenic retrieval solution at $99^{\circ} \mathrm{C}$ in a warm bath (EnVision Flex Target Retrieval solutions, high and low pH, Dako). Endogenous peroxidase was inhibited with a hydrogen peroxide phosphate-buffered solution (EnVision Flex Peroxidase Blocking Reagent, Dako). After incubation with the primary antibodies (ST6Gal-I - rabbit polyclonal, dilution 1:400, pH 6, Cliniscience ${ }^{\circledR}$; ST6Gal-II - rabbit polyclonal, dilution 1:300, pH 6, Cliniscience ${ }^{\circledR}$; ST6GalNac-I - rabbit polyclonal, dilution 1:500, pH 6, Novus ${ }^{\circledR}$; CD4 - 4B12 clone, RTU, pH 9, Dako®; CD8 - C8/144B clone, RTU, pH 9, Dako®, and Granzyme B - GrB-7 clone, dilution 1:20, pH 9, Dako®), the immunological reaction was detected with a polymer dextran coupled with secondary antibody and peroxidase for $15 \mathrm{~min}$ (EnVision Flex HRP, Dako) and diaminobenzidine for 10 
min (EnVision DAB + chromogen, Dako). Counterstaining was performed with hematoxylin for $10 \mathrm{~min}$ (EnVision Flex hematoxylin, Dako). Negative controls were obtained using mouse or rabbit IgG1 (Universal Negative Control Mouse or Universal Negative Control Rabbit, Dako) diluted 1:100, in place of the primary antibodies. MDA-MB231 and MCF7 breast cancer culture cells were known to express ST6GalI, ST6Gal-II and ST6GalNac-I. ${ }^{11}$ From MDA-MB231 and MCF7 cells, we performed cell blocks as positive controls. Methods were previously described. ${ }^{12}$

\section{Immunostaining quantification}

The staining results were evaluated by $\mathrm{CG}$ and $\mathrm{CM}$ based on the intensity and percentage of staining tumor cells, and agreement was reached.

The parametric results were edited as a score by the addition of intensity $(0=$ none, $1=$ weak, $2=$ intermediated, $3=$ strong) and the percentage of tumor cells ( $0=$ none, $1=1 \%$, $2=2 \%$ to $10 \%, 3=11 \%$ to $33 \%, 4=34 \%$ to $66 \%$ and $5>$ $66 \%$ to $100 \%$ ). The range of scores was 0 to 8 . Nonparametric results were calculated as positive for a score $>5$.

Lymphocytes were counted in one hot spot of lymphocytes close to the tumor cells in a high-power field (HPF) and expressed as the number per HPF (400× magnification).

\section{Statistics}

The results were expressed as means and standard error. Mann-Whitney and Spearman's correlation rank tests were performed. A $P$-value $<0.05$ was considered significant. WinSTAT ${ }^{\circledR}$ version 2012 (Fitch Software, Bad Krozinger, Germany) and Excel 2013 (Microsoft Corporation, Redmond, WA, USA) programs were used for statistical analysis.

\section{Results}

\section{TIL subsets in BCs}

We first determined the molecular subtypes of BC TIL subsets, including CD4, CD8 and CTL/NK (granzyme B-positive) (Figure 1). The results reveal that cytotoxic lymphocytes were significantly increased in $\mathrm{TN}$ compared to $\operatorname{LUM}(\mathrm{LUM}=1.5 \pm 2.2 ; \mathrm{HER} 2=4.8 \pm 7.5$ and $\mathrm{TN}=9.6$ \pm 9.9 lymphocytes/HPF; $P<0.0001$ only for LUM versus $\mathrm{TN})$. Although no statistically significant increase in CD4 $(\mathrm{LUM}=18.4 \pm 9.5 ; \mathrm{HER} 2=11.1 \pm 15.0$ and $\mathrm{TN}=14.4 \pm$ 16.6 lymphocytes/HPF) or CD8 (LUM $=22.5 \pm 23.1$; HER2 $=19.5 \pm 27.6$ and $\mathrm{TN}=28.7 \pm 28.5$ lymphocytes $/ \mathrm{HPF}$ ) was observed in all molecular BC subgroups, we noted that CD8 was the predominant T-cell population.

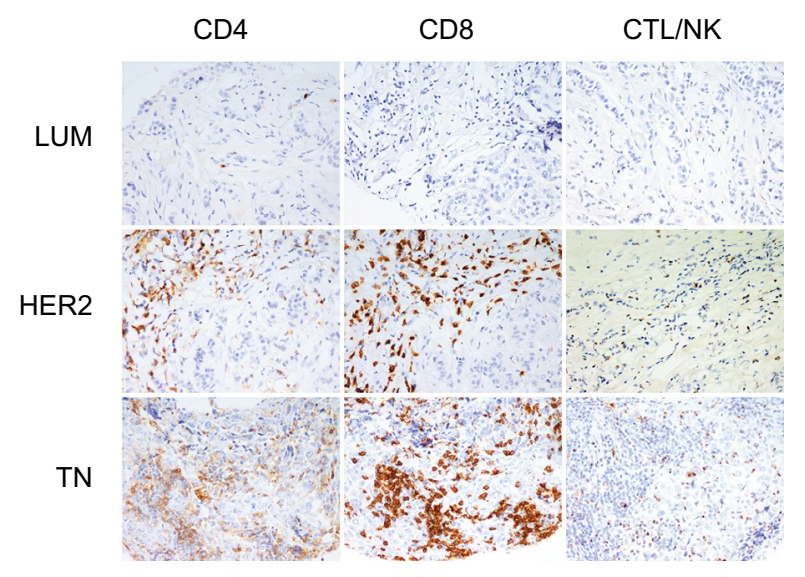

Figure I Tumor-infiltrating lymphocyte (TIL) subsets in different breast cancer groups.

Notes: CD4, CD8 and granzyme-B peroxidase-positive immunohistochemical staining in LUM, HER2 and TN breast cancers: LUM shows a low TIL level in comparison with HER2 or TN. CD8 is the predominant T-cell population. LUM, HER2 and TN are the same case of Figure 2. (200× magnification).

Abbreviations: LUM, luminal; HER2, human epidermal growth factor receptor 2; TN, triple negative; CTL/NK; cytotoxic T lymphocytes/natural killer lymphocytes.

\section{Correlation between TIL and ST6Gal-I}

We next evaluated whether ST6Gal-I influenced the TILs in different BCs (Figures 2 and 3). We found that ST6Gal-I expression was significantly lower in TN than in LUM or HER2 (TN: $2.1 \pm 3.0$ versus LUM: $7.2 \pm 0.99, P<0.0001$ and TN versus HER2: $6.4 \pm 2.3, P<0.0001)$. Concerning TILs, low levels of ST6Gal-I were correlated with an increase in all lymphocytes subsets only for HER2, with no changes observed in LUM or TN. Only in HER2, Spearman's correlations were statistically positive and inverse between ST6Gal-I and CD4 $(r=-0.5 ; P=0.04)$ or CD8 ( $r=0.59 ; P=0.016)$. We conclude that ST6Gal-I plays an important role in HER2 and negatively influences the TIL. Consequently, overexpression of ST6Gal-I in HER2 led to reduced TILs.

\section{Correlation between TILs and ST6Gal-II}

We next characterized ST6Gal-II and found that its expression was similar in all 3 BC groups (LUM: $5.9 \pm 1.5$; HER2: 6.6 \pm 0.6 ; TN: $5.8 \pm 1.8 ; P=$ ns) (Figures 2 and 4). Concerning the amount of TILs, we noted that a low expression level of ST6Gal-II was significantly correlated with a decrease in TILs, mainly in the TN group. Only in TN, Spearman's correlations were statistically positive between ST6Gal-II and CD4 $(r=0.36 ; P=0.006)$ or $\operatorname{CD} 8(r=0.41 ; P=0.0026)$ or CTL $(r=0.28 ; P=0.02)$.

To our knowledge, this is the first time that ST6Gal-II has been described, in situ, in BC. 


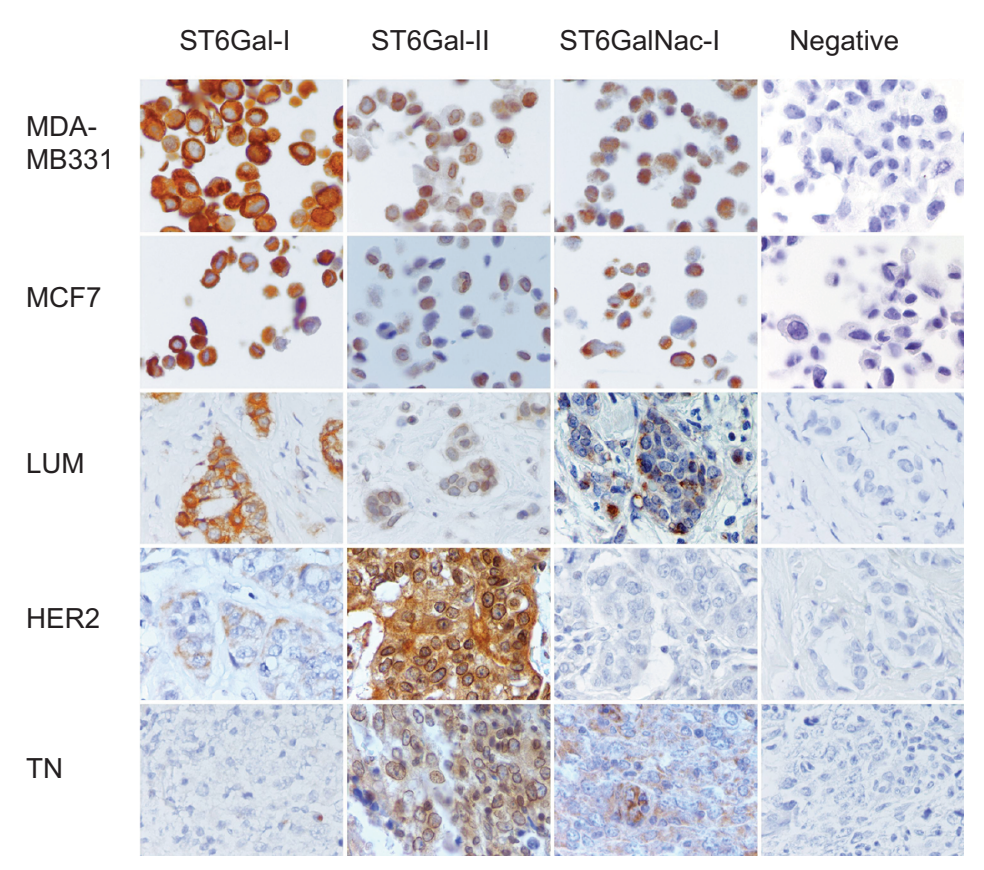

Figure 2 Immunohistochemical expression of ST6Gal-I, ST6Gal-II and ST6GalNac-I.

Notes: Peroxidase-positive immunohistochemical staining in breast carcinomas: MDA-MB23I and MCF7 controls are positive for ST6Gal-I, ST6Gal-II and ST6GalNac-I. ST6Gal-I presents a high expression level in LUM. ST6Gal-II and shows a high expression in TN and HER2. ST6GalNac-I is expressed more in TN. ST6Gal-I shows diffuse cytoplasmic expression, ST6Gal-II shows diffuse cytoplasmic and perinuclear expression and ST6GalNac-I shows granular cytoplasmic positivity. LUM, HER2 and TN are the same case of Figure I. Negative controls were performed with IgGI negative control mouse or rabbit (Dako $\left.{ }^{\circledR}\right)(400 \times$ magnification).

Abbreviations: LUM, luminal; HER2, human epidermal growth factor receptor 2; TN, triple negative; IgGI, immunoglobulin GI.

\section{Correlation between TILs and ST6GalNac-I}

ST6GalNac-I is the enzyme associated with the sialylation of Thomsen-nouvelle antigen (Tn), which is localized on the external glycosylated region of MUC1-VNTR (Figures 2 and 5). ${ }^{13}$ In our previous study, we demonstrated increased expression of MUC1 in LUM compared to TN. ${ }^{9}$ Here, we observed a difference in expression between LUM and HER2 BCs (2.8 \pm 2.7 versus $4.6 \pm 2.9$, respectively; $P=0.02)$ and TN ( $4.6 \pm$ 2.5 respectively; $P=0.002$ ). However, we found only a Spearman's correlation between ST6GalNac-I and CD4 for TN $(\mathrm{r}=$ $0.36 ; P=0.007$ ), suggesting that modulation of this sialylation pathway of MUC1 is not sufficient to have influence on TILs.

\section{Discussion}

Sialylation of tumor cells is complex, involving numerous enzymes that play a major role in oncogenesis and associated processes, such as adhesion, migration, apoptosis and receptor regulation. However, this mechanism of sialylation is not well studied, likely due to the absence of sufficient laboratory tools or complicated investigation techniques. To date, immunohistochemistry, which is used in the present study, is still the primary investigational method of choice. ${ }^{5}$
Here, we evaluated the relationship between $\alpha-2,6$ sialylation and intratumoral T-cell lymphocytes. We found that sialylation could modulate the TILs and that this cell signaling pattern was altered depending on the molecular signature of the BC. Our observations appear particularly relevant in TN for ST6Gal-II and in HER2 for ST6Gal-I.

Moreover, clinical practice has previously described the importance of TILs in the prognosis or therapeutic response of breast cancers. TILs are heterogeneous lymphocyte subsets that are highly variable in different BC subgroups. Previously, TN and HER2 were found to exhibit increased TILs, which were correlated with better overall survival and better chemotherapeutic responses., ${ }^{2,1415}$. Interestingly, we also observed that ST6Gal-I and ST6Gal-II influenced TILs only in HER2 and TN tumors.

Therefore, we propose that the sialyl charge of the membrane glycoproteins could regulate the immune response. First, ST6Gal-I and ST6Gal-II are involved in the $\alpha-2,6$ sialylation of numerous cell membrane glycoproteins, including EGFR, CD45, $\beta 1$ integrin, PECAM, Fas and immunoglobulin. ${ }^{16}$ Second, it has been shown in B16 murine melanoma cell cultures that hypersialylation influences tumor growth and immune escape by alteration of T-cell activity, particularly NK cells, by inhibition of 

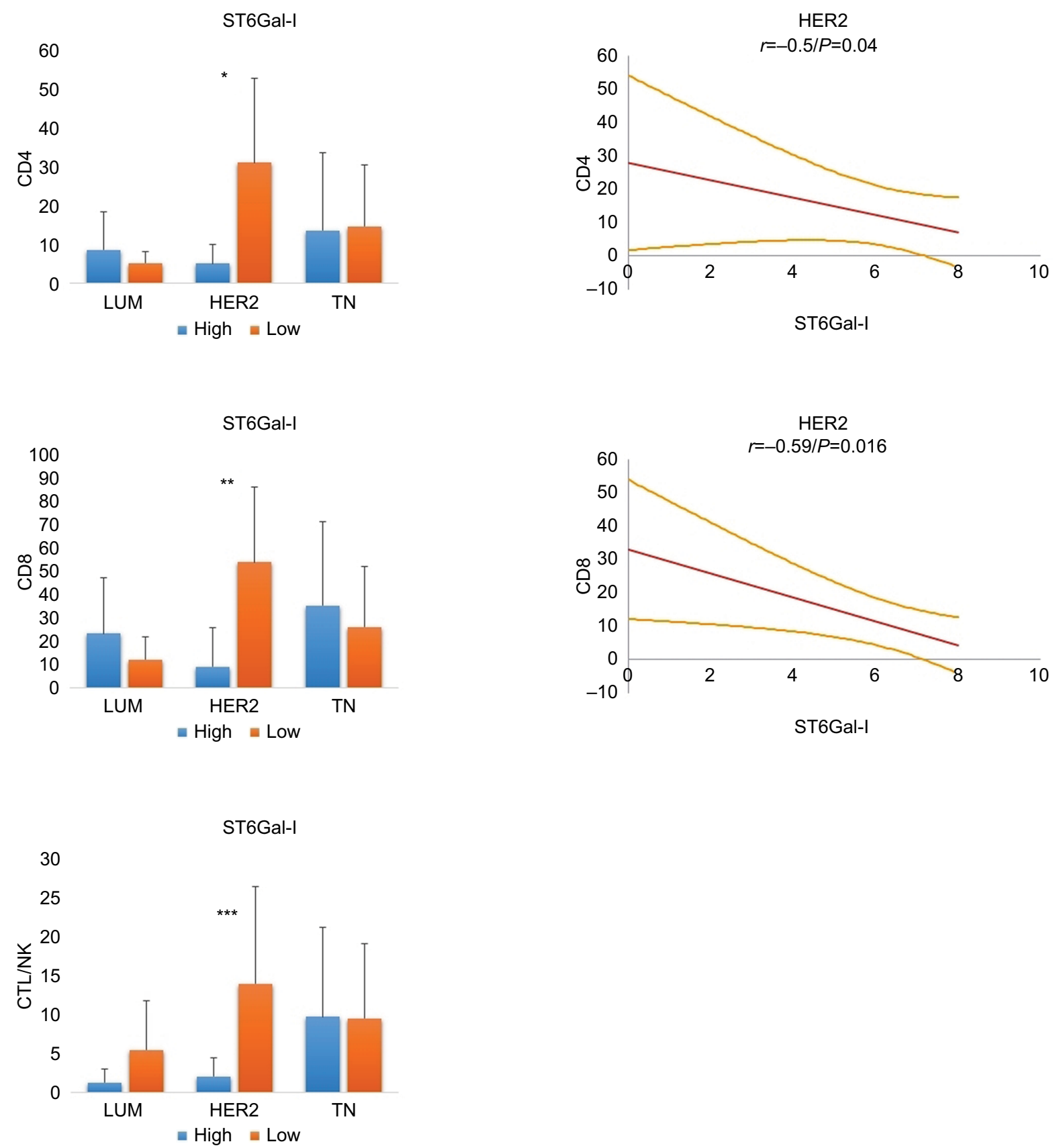

Figure 3 Relationship between ST6Gal-I and TILs in different breast cancer subtypes.

Notes: In HER2 tumors, low levels of ST6Gal-I are correlated with an increase in all lymphocyte subsets $\left({ }^{*} \mathrm{CD} 4: P=0.01 ; * * C D 8: P=0.02 ; * * * C T L / N K: P=\right.$ ns). On the right, statistical negative Spearmen's correlations are documented for CD4 and CD8 positive T lymphocytes in HER2 breast carcinoma (regression line and $95 \%$ Cl). For LUM and TN, we found no significant correlation. We conclude that high expression of ST6Gal-I in HER2 leads to reduced TILs.

Abbreviations: TILs, tumor-infiltrating lymphocytes; HER2, human epidermal growth factor receptor 2; CTL/NK; cytotoxic T lymphocytes/natural killer lymphocytes; LUM, luminal; TN, triple negative; ns, not significant.

DC maturation. ${ }^{17}$ Sialylation has also been associated with other immune mechanisms, such as the sialic acid-binding immunoglobulin-like lectins (siglecs), which are a family of receptors linking cancers cells to immune cells. Moreover, Hudak et $\mathrm{al}^{16}$ demonstrated that increasing global sialylated glycans on cancer cells inhibit human NK activation through the recruitment of siglec. ${ }^{6,18}$

Finally, ST6GalNac-I is an enzyme associated with the sialylation of Tn antigen localized on the external glycosylated region of MUC1-VNTR. ${ }^{13}$ Moreover, sialyl-Tn is known 


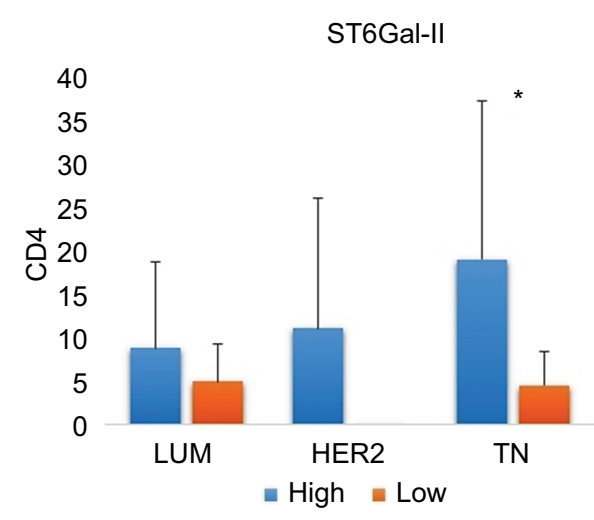

ST6Gal-II

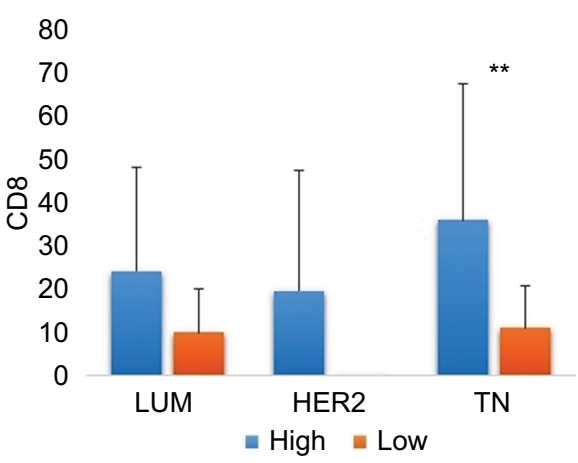

ST6Gal-II

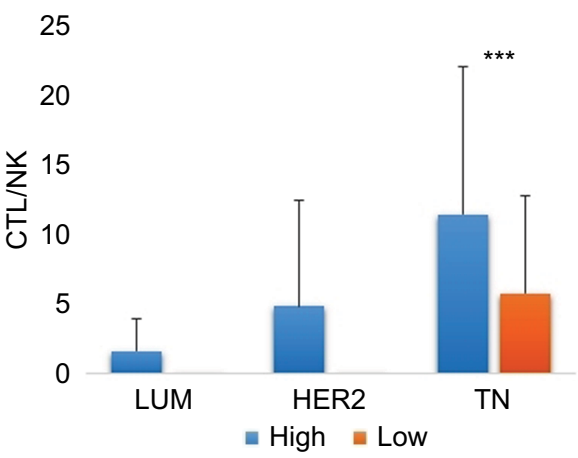

Figure 4 Relationship between ST6Gal-II and TILs in different breast cancer subtypes.

Notes: In TN, low levels of ST6Gal-II are significantly correlated with a decrease in lymphocyte subsets $(* C D 4: P=0.01 ; * * C D 8: P=0.004 ; * * * C T L / N K: P=$ ns). On the right, statistical positive Spearmen's correlations are documented for CD4 and CD8 positive T lymphocytes in TN breast carcinoma (regression line and $95 \% \mathrm{Cl}$ ). For LUM and HER2, we found no significant correlation. We conclude that a loss of expression of ST6Gal-II in TN leads to reduced TILs.

Abbreviations: TILs, tumor-infiltrating lymphocytes; TN, triple negative; CTL/NK; cytotoxic T lymphocytes/natural killer lymphocytes; LUM, luminal; HER2, human epidermal growth factor receptor 2; ns, not significant.

to play a role in the cellular immunologic regulation of tumor cells. ${ }^{18}$ It has also been reported that aberrant glycosylation of MUC1 modulates the immunologic environment through the sialic acid and sialic acid-binding immunoglobulin-like lectin (siglec) pathway. ${ }^{19,20}$ In our study, the relationship between ST6GalNac-I and TIL was not obvious, but the low levels of

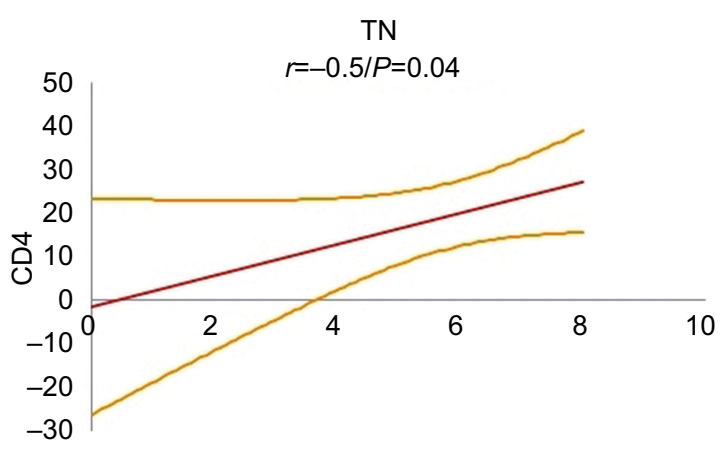

ST6Gal-II

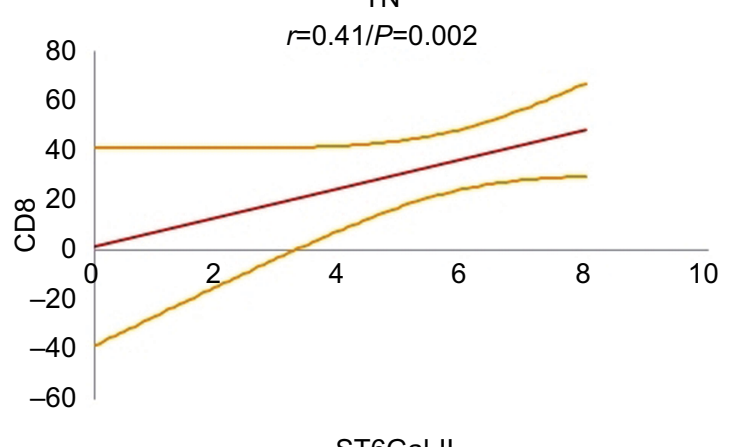

ST6Gal-II
CTL/NKs in LUM or HER2, which broadly express MUC1 compared to TN (no significant difference between LUM and HER2, data not shown), suggest the loss of ST6GalNac-I sialylation activity due to the absence of MUC1 as a substrate. Similarly, EGFR, which is a substrate of ST6Gal-I and ST6Gal-II, is less expressed in LUM or HER2 tumors than 


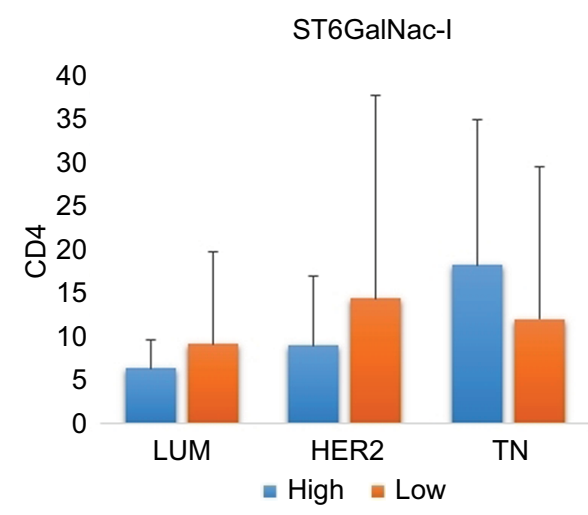

ST6GalNac-I

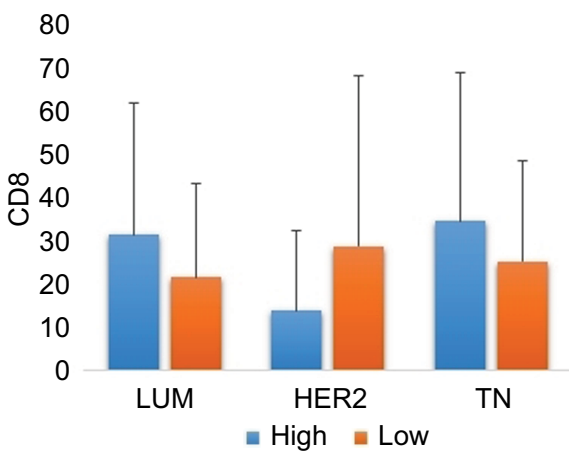

TN

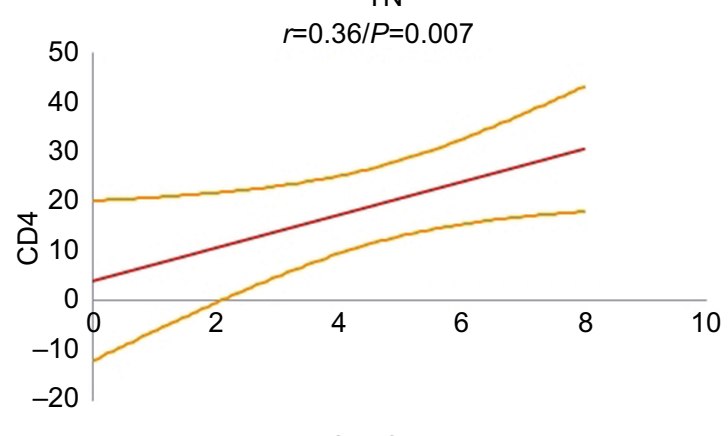

ST6GalNac-I

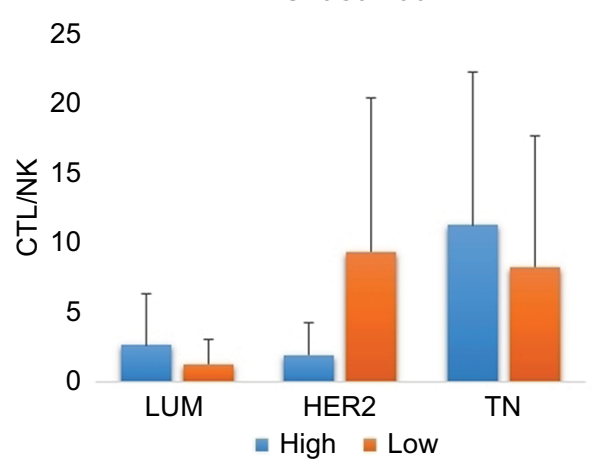

Figure 5 Relationship between ST6GalNac-I and TILs in different breast cancer subtypes.

Notes: On the right, statistical positive Spearmen's correlation is only documented for CD4 positive T lymphocytes in TN breast carcinoma (regression line and 95\% Cl). For ST6GaINac-I, no other significant correlation was observed, suggesting that ST6GalNac-I does not have a major effect on TILs.

Abbreviations: TN, triple negative; TILs, tumor-infiltrating lymphocytes; CTL/NK; cytotoxic T lymphocytes/natural killer lymphocytes.

in TN tumors (no significant difference between LUM and HER2, data not shown), which likely explains the different influences of these enzymes on TILs following molecular BC classification. ${ }^{9}, 12$ Other sialylated membrane glycoproteins or others sialylation enzymes could also be involved, such as ST3Gal or ST6GalNac-II, which also use MUC1 as a substrate. ${ }^{21,22}$

Concerning the relationship between therapeutic resistance or prognosis and sialyltransferases, the most studied enzyme in the literature is ST6Gal-I. Interestingly, in our series, we observed that the loss of ST6Gal-I was associated with a poor prognosis in TN (5-year disease-free survival: $91 \%$ for high expression versus $76 \%$ for low expression, $P=0.04$; data no shown). Park et al demonstrated that loss of ST6Gal-I enhanced EGFR phosphorylation, which was also correlated with the sialylation of EGFR. These authors also showed that the overexpression of ST6Gal-I decreased the effects of gefitinib (an EGFR inhibitor). ${ }^{10}$ Additionally, several authors have demonstrated the colocalization of
MUC1 and EGFR at both the cell membrane and in the nucleus, involving internalization of EGFR and its activation. ${ }^{23,24}$ Moreover, it is known that both MUC1 and EGFR show severe alterations of their glycosylation patterns, which are correlated with the tumor's capacity for metastasis. ${ }^{25}$ It has been demonstrated that ST6Gal-I is associated with chemoresistance to docetaxel in hepatocarcinoma and to gemcitabine in pancreatic ductal adenocarcinoma. ${ }^{26,27}$ Curiously, EGFR is also described as highly glycosylated, and its sialylation confers resistance to tyrosine kinase inhibitors in lung carcinoma cell lines. ${ }^{28,29}$ To our knowledge, we report, for the first time, an inverse relationship between TILs and ST6Gal-I in HER2 tumors. As discussed, the high levels of TILs in HER 2 tumors are associated with a good prognosis. However, MUC4, a highly glycosylated glycoprotein that can also be sialylated, is also associated with trastuzumab (an HER2 inhibitor) resistance ${ }^{30,31}$ Considering these observations, we hypothesize that the sialylation patterns of MUC1, EGFR and likely other membrane glycoproteins could serve as a 
regulatory signal between TILs and the tumor cells and could be one of the pathways underlying therapeutic resistance.

To date, this is the first study to describe ST6Gal-II expression in human $\mathrm{BC}$ in situ. We demonstrated that ST6Gal-II is correlated with TILs in TN tumors. Only one additional report, in bovine breast epithelial cell cultures, has found that ST6Gal-II stimulates pro-inflammatory cytokines, particularly IL-6, known to be active in BCs, therefore suggesting that sialyltransferases could play an important role in the tumor immune microenvironnment. ${ }^{32}$ In human bronchial mucosa and pancreatic cancer cell lines, IL-6 has been shown to increase the expression of ST6Gal-I and ST6Gal-II respectively, suggesting regulation by the immune system. ${ }^{33}$

\section{Conclusion}

Sialylation, mediated by different sialyltransferases, is one of the most important and complex mechanisms involving cell surface receptors and the relationship between the immune microenvironment and the tumor cells. It is not surprising that sialyltransferases are associated with therapeutic resistance, likely by inhibition of the immune response.

\section{Disclosure}

The authors report no conflicts of interest in this work.

\section{References}

1. Perou CM. Molecular stratification of triple-negative breast cancers. Oncologist. 2011;16(Suppl 1):61-70.

2. Miyan M, Schmidt-Mende J, Kiessling R, Poschke I, de Boniface J. Differential tumor infiltration by T-cells characterizes intrinsic molecular subtypes in breast cancer. J Transl Med. 2016;14:227.

3. Mao Y, Qu Q, Chen X, Huang O, Wu J, Shen K. The prognostic value of tumor-infiltrating lymphocytes in breast cancer: A systematic review and meta-analysis. PLoS One. 2016;11(4):e0152500.

4. Vajaria BN, Patel PS. Glycosylation: A hallmark of cancer? Glycoconj J. 2017;34(2):147-156

5. Schultz MJ, Swindall AF, Bellis SL. Regulation of the metastatic cell phenotype by sialylated glycans. Cancer Metastasis Rev. 2012;31(3-4): $501-518$.

6. Lu J, Gu J. Significance of $\beta$-galactoside $\alpha 2,6$ sialyltranferase 1 in cancers. Molecules. 2015;20(5):7509-7527.

7. Wreschner DH, McGuckin MA, Williams SJ, et al. Generation of ligand-receptor alliances by "SEA" module-mediated cleavage of membrane-associated mucin proteins. Protein Sci. 2002;11(3): 698-706.

8. Lillehoj EP, Hyun SW, Feng C, et al. NEU1 sialidase expressed in human airway epithelia regulates epidermal growth factor receptor (EGFR) and MUC1 protein signaling. J Biol Chem. 2012;287(11):8214-8231.

9. Garbar C, Mascaux C, Giustiniani J, et al. Autophagy is decreased in triple-negative breast carcinoma involving likely the MUC1-EGFRNEU1 signalling pathway. Int J Clin Exp Pathol. 2015;8(5):4344-4355.

10. Park JJ, Yi JY, Jin YB, et al. Sialylation of epidermal growth factor receptor regulates receptor activity and chemosensitivity to gefitinib in colon cancer cells. Biochem Pharmacol. 2012;83(7):849-857.
11. Ma X, Dong W, Su Z, et al. Functional roles of sialylation in breast cancer progression through miR-26a/26b targeting ST8SIA4. Cell Death Dis. 2016;7(12):e2561.

12. Garbar C, Mascaux C, Giustiniani J, Merrouche Y, Bensussan A. Chemotherapy treatment induces an increase of autophagy in the luminal breast cancer cell MCF7, but not in the triple-negative MDA-MB231. Sci Rep. 2017;7(1):7201.

13. Madsen CB, Lavrsen K, Steentoft C, Vester-Christensen MB, Clausen $\mathrm{H}$, Wandall HH, Pedersen AE. Glycan elongation beyond the mucin associated Tn antigen protects tumor cells from immune-mediated killing. PLoS One. 2013;8(9):e72413.

14. Loi S, Sirtaine N, Piette F, et al. Prognostic and predictive value of tumor-infiltrating lymphocytes in a Phase III randomized adjuvant breast cancer trial in node-positive breast cancer comparing the addition of docetaxel to doxorubicin with doxorubicin-based chemotherapy: BIG 02-98. J Clin Oncol. 2013;31(7):860-867.

15. Luen SJ, Salgado R, Fox S, et al. Tumour-infiltrating lymphocytes in advanced HER2-positive breast cancer treated with pertuzumab or placebo in addition to trastuzumab and docetaxel: A retrospective analysis of the CLEOPATRA study. Lancet Oncol. 2017;18(1):52-62.

16. Hudak JE, Canham SM, Bertozzi CR. Glycocalyx engineering reveals a siglec-based mechanism for NK cell immunoevasion. Nat Chem Biol. 2014;10(1):69-75.

17. Perdicchio M, Cornelissen LA, Streng-Ouwehand I, et al. Tumor sialylation impedes $\mathrm{T}$ cell mediated anti-tumor responses while promoting tumor associated-regulatory T cells. Oncotarget. 2016;7(8): 8771-8782.

18. Ogata S, Maimonis PJ, Itzkowitz SH. Mucins bearing the cancerassociated sialosyl-Tn antigen mediate inhibition of natural killer cell cytotoxicity. Cancer Res. 1992;52(17):4741-4746.

19. Beatson R, Tajadura-Ortega V, Achkova D, et al. The mucin MUC1 modulates the tumor immunological microenvironment through engagement of the lectin Siglec-9. Nat Immunol. 2016;17(11): 1273-1281.

20. Tanida S, Akita K, Ishida A, et al. Binding of the sialic acid-binding lectin, Siglec-9, to the membrane mucin, MUC1, induces recruitment of $\beta$-catenin and subsequent cell growth. J Biol Chem. 2013;288(44): 31842-31852.

21. Marcos NT, Pinho S, Grandela C, et al. Role of the human ST6GalNAc-I and ST6GalNAc-II in the synthesis of the cancer-associated sialyl-Tn antigen. Cancer Res. 2004;64(19):7050-7057.

22. Solatycka A, Owczarek T, Piller F, et al. MUC1 in human and murine mammary carcinoma cells decreases the expression of core $2 \beta 1,6-\mathrm{N}$ acetylglucosaminyltransferase and $\beta$-galactoside $\alpha 2,3$-sialyltransferase. Glycobiology. 2012;22(8):1042-1054.

23. Bitler BG, Goverdhan A, Schroeder JA. MUC1 regulates nuclear localization and function of the epidermal growth factor receptor. J Cell Sci. 2010;123(Pt 10):1716-1723.

24. Neeraja Dharmaraj, Engel BJ, Carson DD. Activated EGFR stimulates MUC1 expression in human uterine and pancreatic cancer cell lines. J Cell Biochem. 2013;114(10):2314-2322.

25. Senapati S, Das S, Batra SK. Mucin-interacting proteins: from function to therapeutics. Trends Biochem Sci. 2010 Apr;35(4):236-45.

26. Chen X, Wang L, Zhao Y, et al. ST6Gal-I modulates docetaxel sensitivity in human hepatocarcinoma cells via the p38 MAPK/caspase pathway. Oncotarget. 2016;7(32):51955-51964.

27. Chakraborty A, Dorsett KA, Trummell HQ, et al. ST6Gal-I sialyltransferase promotes chemoresistance in pancreatic ductal adenocarcinoma by abrogating gemcitabine-mediated DNA damage. J Biol Chem. 2018;293(3)984-994.

28. Yen HY, Liu YC, Chen NY, et al. Effect of sialylation on EGFR phosphorylation and resistance to tyrosine kinase inhibition. Proc Natl Acad Sci USA. 2015;112(22):6955-6960.

29. Peiris D, Spector AF, Lomax-Browne H, et al. Cellular glycosylation affects Herceptin binding and sensitivity of breast cancer cells to doxorubicin and growth factors. Sci Rep. 2017;7:43006. 
30. Wimana Z, Gebhart G, Guiot T, et al. N-acetylcysteine breaks resistance to trastuzumab caused by MUC4 overexpression in human HER2 positive BC-bearing nude mice monitored by ${ }^{89} \mathrm{Zr}$-Trastuzumab and ${ }^{18} \mathrm{~F}$-FDG PET imaging. Oncotarget. 2017;8(34):56185-56198.

31. Laporte B, Gonzalez-Hilarion S, Maftah A, Petit JM. The second bovine $\beta$-galactoside- $\alpha 2,6$-sialyltransferase (ST6Gal II): Genomic organization and stimulation of its in vitro expression by IL-6 in bovine mammary epithelial cells. Glycobiology. 2009;19(10):1082-1093.
32. Groux-Degroote S, Krzewinski-Recchi MA, Cazet A, et al. IL-6 and IL-8 increase the expression of glycosyltransferases and sulfotransferases involved in the biosynthesis of sialylated and/or sulfated Lewis ${ }^{\mathrm{X}}$ epitopes in the human bronchial mucosa. Biochem J. 2008;410(1):213-223.

33. Bassagañas S, Allende H, Cobler L, Ortiz MR, Llop E, de Bolós C, Peracaula R. Inflammatory cytokines regulate the expression of glycosyltransferases involved in the biosynthesis of tumor-associated sialylated glycans in pancreatic cancer cell lines. Cytokine. 2015;75(1):197-206.
Cancer Management and Research

\section{Publish your work in this journal}

Cancer Management and Research is an international, peer-reviewed open access journal focusing on cancer research and the optimal use of preventative and integrated treatment interventions to achieve improved outcomes, enhanced survival and quality of life for the cancer patient The manuscript management system is completely online and includes
Dovepress

a very quick and fair peer-review system, which is all easy to use. Visit http://www.dovepress.com/testimonials.php to read real quotes from published authors. 